\title{
Positive association of breastfeeding on respiratory syncytial virus infection in hospitalized infants: a multicenter retrospective study
}

Min Jeong Jang, $\mathrm{MD}^{1}, *$, Yong Joo Kim, MD, $\mathrm{PhD}^{2}{ }^{2}$, Shinhye Hong, $\mathrm{MD}^{2}$, Jaeyoon $\mathrm{Na}, \mathrm{MD}^{2}$, Jong Hee Hwang, $\mathrm{MD}, \mathrm{PhD}^{3}$, Son Moon Shin, $\mathrm{MD}, \mathrm{PhD}^{4}$, Yong Min Ahn, $\mathrm{MD}, \mathrm{PhD}^{1}$

${ }^{1}$ Department of Pediatrics, Nowon Eulji Medical Center, Eulji University, Seoul, Korea; ${ }^{2}$ Department of Pediatrics, Hanyang University Seoul Hospital, Seoul, Korea; ${ }^{3}$ Department of Pediatrics, Inje University Ilsan Paik Hospital, Goyang, Korea; ${ }^{4}$ Department of Pediatrics, Inje University Busan Paik Hospital, Busan, Korea

Background: Breastfeeding reportedly reduces the overall frequency of infections. Respiratory syncytial virus (RSV), the most common respiratory pathogen in infants, involves recurrent wheezing and has a pathogenic mechanism related to airway structural damage.

Purpose: This study aimed to investigate whether breastfeeding has a beneficial effect against RSV-induced respiratory in fection compared to formula feeding among infants in Korea.

Methods: We retrospectively reviewed the medical records of infants under 1 year of age who were admitted with RSV infection between January 2016 and February 2018 at the department of pediatrics of 4 hospitals. We investigated the differences in clinical parameters such as cyanosis, chest retraction, combined infection, fever duration, oxygen use, oxygen therapy duration, intensive care unit (ICU) admission, and corticosteroid treatment of exclusive breast milk feeding (BMF), artificial milk formula fed (AMF), and mixed feeding (MF) groups.

Results: Among the 411 infants included in our study, 94, 161 , and 156 were included in the BMF, MF, and AMF groups, respectively. The rates of oxygen therapy were significantly different among the BMF (4.3\%), MF (8.1\%), and AMF (13.5 $\%)$ groups $(P=0.042)$. The odds ratios $(\mathrm{ORs})$ for oxygen therapy was significantly higher in the AMF group than in the BMF group (adjusted OR, 3.807; 95\% confidence interval, $1.22-11.90 ; P=0.021)$. The ICU admission rate of the BMF group (1.1\%) was lower than that of the MF (3.5\%) and AMF $(4.5 \%)$ groups; however, the dissimilarity was not statistically significant $(P=0.338)$.

Conclusion: The severity of RSV infection requiring oxygen therapy was lower in the BMF than the AMF group. This protective role of human milk on RSV infection might decrease the need for oxygen therapy suggesting less airway damage.

Key words: Breast feeding, Respiratory syncytial virus infection, Infant

\section{Key message}

Question: Human milk has stronger antiviral, antibacterial, and anti-inflammatory properties compared with formula milk. How dose breastfeeding affect respiratory syncytial virus infection in Korea?

Finding: Breastfed infants required less oxygen therapy and possible intensive care unit admission than artificial formulafed infants during respiratory syncytial virus infection.

Meaning: This protective role of breast milk on RSV severity can be a supporting evidence for promoting breastfeeding in Korea.

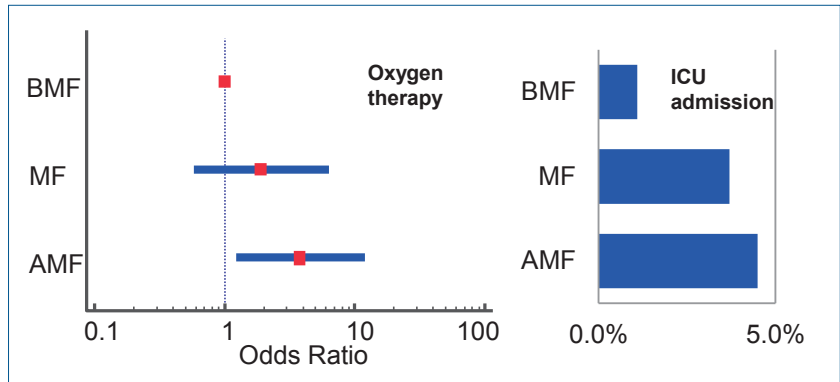

Graphical abstract. Infants with artificial milk formula feeding (AMF) required more oxygen therapy during respiratory syncytial virus lower respiratory infection than infants with breast milk feeding (BMF) (adjusted odds ratios, 3.807; 95\% confidence interval, 1.22-11.90; $P=0.021$ ). Intensive care unit (ICU) admission rate was less among in BMF (1.1\%) than AMF (4.5\%) group but could not get statistical significance.

\section{Introduction}

The policy statement about breastfeeding from the American Academy of Pediatrics (AAP) introduced numerous health benefits of human milk for babies. ${ }^{1)}$ Human milk has stronger antiviral, antibacterial, and anti-inflammatory properties compared with formula milk.2) Ip et al. ${ }^{3)}$ reported the risk of hospitalization for lower respiratory tract infections during the first year

\footnotetext{
Corresponding author: Young Min Ahn, MD, PhD. Department of Pediatrics, Nowon Eulji Medical Center, Eulji University, 68, Hangeulbiseok-ro, Nowon-gu, Seoul 01830, Korea 凶E-mail: aym3216@eulji.ac.kr, https://orcid.org/0000-0002-1697-8041

*These authors contributed equally to this study as co-first authors.

Received: 30 April, 2019, Revised: 4 November, 2019, Accepted: 11 November, 2019

This is an open-access article distributed under the terms of the Creative Commons Attribution Non-Commercial License (http://creativecommons.org/licenses/bync/4.0/) which permits unrestricted non-commercial use, distribution, and reproduction in any medium, provided the original work is properly cited. 
of life is reduced to $72 \%$ if infants are exclusively breastfed for more than 4 months.

Respiratory syncytial virus (RSV) infection is nearly universal among children by their second birthday and asymptomatic RSV infection is unusual in young infants. So the hospitalization rate is about $0.5 \%-4 \%$ under the common diagnosis of bronchiolitis and pneumonia although two is often indistinguishable and frequently coexists. ${ }^{4)}$ It is known that the severity of RSV bronchiolitis may be related to chronic lung disease, congenital heart disease, immunodeficiency, low birth weight and prematurity. ${ }^{5)}$ Other factors associated with RSV infection include winter births, poverty, malnutrition, tobacco smoke exposure, lack of breast feeding and multiple gestations. ${ }^{6}$ ) Furthermore, there were many studies on the increased risk of recurrent wheezing and asthma in the later life of a patient who has suffered from RSV-induced bronchiolitis. ${ }^{7,8)}$ Long-term pulmonary maturity might be threatened if young infants contracts severe RSV bronchiolitis. ${ }^{9)}$ Specific treatment or vaccine against RSV is not yet available; supportive care including hydration and respiratory support can be provided in the hospitals. ${ }^{10)}$ Palivizumab, which can be used under specific indications due to high cost, has some evidence for prevention and reducing recurrent wheezing among nonatopic children to support the pathogenesis of RSV that damages airway. ${ }^{11)}$ Thus, effective intervention for severity is urgently needed for high risk infants, especially those aged less than 6 months, to reduce mortality, recurrent wheezing and subsequent asthma. ${ }^{12)}$

There have been many efforts to find out which could protect infants from the sequelae or mortality by RSV. Human milk feeding was proved to be one of the effective protectors. Nishimura et al. ${ }^{13)}$ reported that breastfeeding could reduce the severity of RSV infection among the young infants via multicenter study.

Many women in Korea might know the benefits of breast milk; however, they do not seriously consider the long-term benefits and easily give up breastfeeding. Thus, the rate of exclusive breastfeeding in Korea is low. ${ }^{14)}$ Additionally, in the real- world setting, there are no clinical studies on the positive effect of human milk among infants in Korea. Therefore, in this study we investigated whether breastfeeding compared to formula feeding has a beneficial effect against RSV bronchiolitis in young infants.

\section{Methods}

\section{Study design}

The presence of RSV bronchiolitis was confirmed either by real-time quantitative-polymerase chain reaction (probe hybridization using Anyplex II RV16 Detection v1.1, Seegene, Inc., Seoul, Korea) or rapid antigen detection test (Alere BinaxNOW RSV, Seegene, Inc.) using a nasopharyngeal swab. Infants aged less than 12 months were included in the study; neonates and preterm babies and infants with underlying diseases were excluded. We retrospectively reviewed the medical records of infants who were admitted due to RSV-induced respiratory illness between January 2016 and February 2018 in the department of pediatrics of 4 hospitals, namely, Nowon Eulji Medical Center, Hanyang University Seoul Hospital, Inje University Ilsan Paik Hospital, and Dankook University Cheil General Hospital. This study was approved by the Institutional Review Board (IRB) of Nowon Eulji Medical Center (IRB No. 2017-03-013). Informed consent was waived by the IRB.

We divided the infants into 3 groups based on their feeding patterns: breast milk feeding (BMF), mixed feeding (MF), and artificial milk formula (AMF) groups. We investigated whether sex, birth weight, presence of older sibling, weight at admission, age at admission, and past medical histories influenced the association of feeding patterns on infection contraction. We reviewed medical records of all patients who were included. Additionally, we studied the impact of the different feeding patterns on cyanosis, chest retraction, combined infection, duration of fever, oxy. gen use, duration of oxygen therapy, intensive care unit (ICU) admission and duration of stay, and corticosteroid treatment.

Table 1. Patients' baseline characteristics

\begin{tabular}{|c|c|c|c|c|c|}
\hline Variable & Total population $(n=411)$ & $\operatorname{BMF}(n=94,22.9 \%)$ & MF $(n=161,39.2 \%)$ & AMF $(n=156,37.0 \%)$ & $P$ value \\
\hline Sex & & & & & 0.088 \\
\hline Male & $240(58.4)$ & $51(54.3)$ & $92(57.1)$ & $97(62.2)$ & \\
\hline Female & 171 (41.6) & $43(45.7)$ & $69(42.9)$ & $59(37.8)$ & \\
\hline Birth weight (kg) & $3.22(2.95-3.50)$ & $3.23(2.90-3.46)$ & $3.20(2.90-3.52)$ & $3.23(3.00-3.52)$ & 0.787 \\
\hline Gestational age (wk) & $39(38-40)$ & $39(38-40)$ & $39(38-40)$ & $38(38-40)$ & 0.108 \\
\hline Siblings & & & & & 0.089 \\
\hline Yes & $238(59.2)$ & $60(65.2)$ & $82(52.6)$ & $96(62.3)$ & \\
\hline No & $164(40.8)$ & $32(34.8)$ & $74(47.4)$ & $58(37.3)$ & \\
\hline Weight at admission (kg) & $7.90(6.10-9.20)$ & $8.15(6.13-9.51)$ & $7.50(5.80-8.73)$ & $8.25(6.25-9.50)$ & 0.031 \\
\hline Age at admission (mo) & $5(2-9)$ & $6(2-9)$ & $4(2-7)$ & $6(3-9)$ & 0.011 \\
\hline Past disease history & & & & & 0.094 \\
\hline Yes & $30(7.3)$ & $5(5.3)$ & $8(5.0)$ & 17 (10.9) & \\
\hline No & 381 (92.7) & $89(94.7)$ & 153 (95.0) & 139 (89.1) & \\
\hline
\end{tabular}

Values are presented as number (\%) or median (interquartile range) ${ }^{\mathrm{b})}$.

BMF, breast milk fed group; MF, mixed (breast milk and formula) fed group; AMF, artificial milk formula fed group.

${ }^{a)}$ Chi-square test. ${ }^{b}$ Kruskal-Wallis test. 
As several patients did not have the information, family history of allergy and asthma could not be included. Pulse oxygen saturation was monitoring if respiratory failure symptoms such as chest retraction, nasal flaring, and cyanosis were seen. Applying oxygen started when pulse oxygen saturation was less than $90 \%$ according to bronchiolitis guideline from AAP. ${ }^{15)}$

\section{Statistical analysis}

Numerical quantitative data are presented as median (interquartile range) or median (range) and were analyzed using the Kruskal-Wallis test or Jonckheere trend test. Categorical data are presented as frequency (\%) and were analyzed using the chisquare test, chi-square trend test, or Fisher exact test.

Odds ratios (ORs) were estimated by analyzing the logistic regression models. The crude OR and the adjusted OR were estimated by univariate and multivariate logistic regression models, respectively. Statistical analyses were performed using IBM SPSS Statistics ver. 22.0 (IBM Co., Armonk, NY, USA). $P<0.05$ were considered to be statistically significant.

\section{Results}

There were some differences among BMF, MF, and AMF groups in terms of body weight and the age at the admission. The infants in the MF group (7.50 kg; 4 months old) weighed less and were younger than those in the BMF (7.90 kg; 5 months) and AMF groups ( $8.25 \mathrm{~kg} ; 6$ months). However, there were no differences between the groups in terms of sex, birth weight, gestational period, sibling, and past medical history (Table 1). Past diseases of 30 infants were very diverse, including neonatal jaundice, gastroenteritis, and asymptomatic congenital heart disease.

The rate of infants underwent oxygen therapy was significantly different among BMF (4.3\%), MF (8.1\%), and AMF (13.5\%) groups $(P=0.042)$. There also existed a significant increasing trend of the rate of oxygen therapy by changing groups from $\mathrm{BMF}$ to MF and from MF to AMF ( $P$ value for trend $=0.012$ ). The ORs for oxygen therapy are significantly higher in AMF groups than BMF group (adjusted OR, 3.807; 95\% confidence interval, 1.22-11.90; $P=0.021)$. The ICU admission rate in the BMF group (1.1\%) was observed to be lower than that in the MF group (3.5\%) and AMF group (4.5\%); however, the dissimila-

Table 2. Patients' clinical outcomes

\begin{tabular}{|c|c|c|c|c|c|c|}
\hline \multirow{2}{*}{ Variable } & \multirow{2}{*}{ Total population $(n=411)$} & \multirow{2}{*}{ BMF $(n=94)$} & \multirow{2}{*}{$M F(n=161)$} & \multirow{2}{*}{$\operatorname{AMF}(n=156)$} & \multicolumn{2}{|c|}{$P$ value } \\
\hline & & & & & Difference & Trend \\
\hline Hospitalization period (day) & $4(1-23)$ & $4(2-15)$ & $4(1-16)$ & $4(1-23)$ & 0.189 & 0.111 \\
\hline Cyanosis & & & & & 0.488 & 0.331 \\
\hline Yes & $7(1.7)$ & $2(2.1)$ & $4(2.5)$ & $1(0.6)$ & & \\
\hline No & $404(98.3)$ & $92(97.9)$ & $157(97.5)$ & $155(99.4)$ & & \\
\hline Chest retraction & & & & & 0.76 & 0.634 \\
\hline Yes & $62(15.1)$ & $12(12.8)$ & $26(16.1)$ & $24(15.4)$ & & \\
\hline No & $349(84.9)$ & $82(87.2)$ & $135(83.9)$ & $132(84.6)$ & & \\
\hline Coinfection $(n=368)$ & & & & & 0.417 & 0.263 \\
\hline Yes & $97(26.4)$ & $16(20.5)$ & $41(27.7)$ & $40(28.2)$ & & \\
\hline No & $271(73.6)$ & $62(79.5)$ & $107(72.3)$ & $102(71.8)$ & & \\
\hline Fever & & & & & 0.039 & 0.755 \\
\hline Yes & $236(57.4)$ & $59(62.8)$ & $80(49.7)$ & $97(62.2)$ & & \\
\hline No & $175(42.6)$ & $35(37.2)$ & $81(50.3)$ & $59(37.8)$ & & \\
\hline Duration & $1(0-10)$ & $2(0-7)$ & $0(0-9)$ & $2(0-10)$ & 0.054 & 0.631 \\
\hline Oxygen therapy & & & & & 0.042 & 0.012 \\
\hline Yes & $38(9.2)$ & $4(4.3)$ & $13(8.1)$ & $21(13.5)$ & & \\
\hline No & $373(90.8)$ & $90(95.7)$ & $148(91.9)$ & $135(86.5)$ & & \\
\hline Duration & $0(0-15)$ & $0(0-7)$ & $0(0-10)$ & $0(0-15)$ & 0.041 & 0.012 \\
\hline ICU admission & & & & & 0.338 & 0.168 \\
\hline Yes & $14(3.4)$ & $1(1.1)$ & $6(3.7)$ & $7(4.5)$ & & \\
\hline No & $397(96.6)$ & $93(98.9)$ & $155(96.3)$ & $149(95.5)$ & & \\
\hline Duration (day) & $0(0-15)$ & $0(0-6)$ & $0(0-6)$ & $0(0-15)$ & 0.359 & 0.192 \\
\hline Corticosteroid treatment & & & & & 0.52 & 0.299 \\
\hline Yes & $83(20.2)$ & $17(18.1)$ & $30(18.6)$ & $36(23.1)$ & & \\
\hline No & $328(79.8)$ & 77 (81.9) & $131(81.4)$ & $120(76.9)$ & & \\
\hline
\end{tabular}

Values are presented as number (\%)a) or median (range ${ }^{\mathrm{b})}$.

BMF, breast milk fed group; MF, mixed (breast milk and formula) fed group; AMF, artificial milk formula fed group; ICU, intensive care unit.

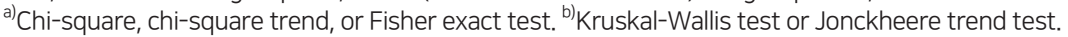

Boldface indicates a statistically significant difference with $P<0.05$. 
Table 3. Univariate and multivariate analyses (outcome=need for 02 therapy)

\begin{tabular}{|c|c|c|c|c|c|c|}
\hline \multirow{2}{*}{ Variable } & \multicolumn{3}{|c|}{ Univariate logistic model } & \multicolumn{3}{|c|}{ Multivariate logistic model ${ }^{a)}$} \\
\hline & Crude OR & $95 \% \mathrm{Cl}$ & $P$ value & Adjusted OR & $95 \% \mathrm{Cl}$ & $P$ value \\
\hline \multicolumn{7}{|l|}{ Sex (ref. female) } \\
\hline Male & 1.102 & $0.557-2.181$ & 0.78 & & & \\
\hline Birth weight & 1.204 & $0.529-2.737$ & 0.658 & & & \\
\hline Gestational age & 0.898 & $0.666-1.21$ & 0.478 & & & \\
\hline \multicolumn{7}{|l|}{ Siblings (ref. no) } \\
\hline Siblings (yes) & 3.235 & $1.385-7.556$ & 0.007 & 2.898 & $1.207-6.957$ & 0.017 \\
\hline Weight at admission & 0.719 & $0.602-0.858$ & $<0.001$ & 0.695 & $0.506-0.954$ & 0.025 \\
\hline Age at admission & 0.872 & $0.784-0.97$ & 0.012 & 1.011 & $0.841-1.215$ & 0.910 \\
\hline \multicolumn{7}{|l|}{ Past history (ref. no) } \\
\hline Clinical history (yes) & 2.727 & $1.039-7.157$ & 0.042 & 2.994 & $1.008-8.893$ & 0.048 \\
\hline Feeding type (BMF) & & & 0.052 & & & 0.039 \\
\hline MF & 1.976 & $0.625-6.247$ & 0.246 & 1.905 & $0.577-6.29$ & 0.290 \\
\hline AMF & 3.500 & $1.163-10.536$ & 0.026 & 3.807 & $1.218-11.898$ & 0.021 \\
\hline
\end{tabular}

$\mathrm{OR}$, odds ratio; $\mathrm{Cl}$, confidence interval; BMF, breast milk feeding group; $\mathrm{MF}$, mixed breast milk and formula feeding group; AMF, artificial milk formula feeding.

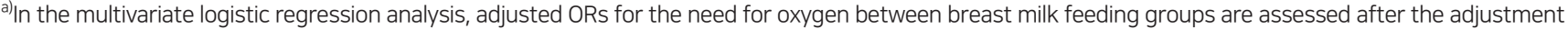
for variables such as siblings, weight at admission, age at admission, and past history, which were significant in the univariate logistic regression analysis.

rity was not statistically significant $(P=0.338)$. There were no significant differences among the groups in admission period, cyanosis, chest retraction, viral coinfection, febrile status, ICU duration, and corticosteroid treatment (Table 2).

Using univariate and multivariate models, we identified the potential risk factors including past medical history, siblings, and body weight at admission that influenced on the need for oxygen administration in infants. After adjusting for the identified risk factors, the association of the different types of feeding on oxygen administration was significant $(P=0.039)$ in multivariable logistic regression (Table 3).

\section{Discussion}

In this study, we found that AMF group needed more oxygen than BMF group, and that breastfeeding was significant in reducing the rate of oxygen application even after adjusting the identified confounding factors. Infants admitted ICU was less than BMF group, but the statistical significance was not found due to the small numbers under ICU care.

Human milk possesses diverse trophic factors which regulate the immune responses. The concentration of lactoferrin in human milk is 1,000 -fold of that in plasma. ${ }^{16}$ ) It decreases the production of chemokines in epithelial cells, ${ }^{17)}$ and reduces chemokine responses by recruiting neutrophils. ${ }^{18)}$ Oligosaccharides and procathepsin $\mathrm{D}$ in human milk alter the neutrophil adhesion molecule CD11b and L-selectin expression, resulting in decreased neutrophil infiltration and activation of airway epithelial cells in viral illness. ${ }^{19)}$

Breast milk makes to increase numbers of Corynebacterium and Dolosigranulum colonies, which are the newly recognized family members of lactic acid bacterium. This is considered to be a healthy microbiota ${ }^{20)}$ to enhance the immunologic response during viral illness. So, human milk has stronger antiviral, anti- bacterial, and anti-inflammatory properties compared with formula milk. ${ }^{2)}$ AAP reaffirms its recommendation of exclusive breastfeeding for about 6 months, followed by continued breastfeeding as complementary foods are introduced, with continuation of breastfeeding for 1 year or longer as mutually desired by mother and infant. ${ }^{1}$

The nationwide trend of breastfeeding rate (BR) in Korea was surveyed among the Organization for Economic Co-operation and Development countries in 2013, and the exclusive BR at the $3 \mathrm{rd}$, the 4th, and the 6th months of life in Korea was 50\%, $40.5 \%$, and $11.4 \%$ respectively. ${ }^{14)}$ Moreover, the government report in 2016 showed that the rate of exclusive BR was 52.6\% at 1 month old, $47.2 \%$ at 3 months old, and rapidly dropped to $9.4 \%$ at 6 months old. ${ }^{21)}$

To reflect the benefits of breast milk best, RSV infection was chosen because it is a common and apparent disease among young infants with high morbidity and mortality rate, but there is no specific treatment despite of frequent wheezing sequale after recovery. According to this study, breastfeeding can reduce applying oxygen for infants with respiratory distress (chest retraction, nasal flaring, and desaturation under 90\%) than artificial formula feeding. When RSV bronchiolitis is exacerbated, airway narrowing is caused by virus-induced necrosis of the bronchiolar epithelium, hypersecretion of mucus, and edema of the surrounding submucosa. Those factors lead to systemic hypoxia. ${ }^{4)}$ In other words, a condition requiring oxygen supplementation in bronchiolitis indicates a higher severity, suggesting that breastfeeding can reduce progression of RSV infection.

Breastfeeding confers protection against both incidence and severity of RSV disease, particularly in those born prematurely, as well as the subsequent development of recurrent wheezing illness. ${ }^{22)}$ The improvement in disease severity correlates with the decrease in concentration of immune-modulator such as airway chemokine, presence of activation markers, and inflammatory cellular infiltrate in exclusively breastfed infants but not in 
those who were partially breastfed. ${ }^{23)}$ Serum interferon (IFN)- $\alpha$ level is markedly higher in breastfed RSV-infected infants than nonbreastfed infants during the course of the disease, as well as 2-4 weeks postinfection. Although previous study showed no difference in IFN- $\alpha$ production between the infants who had been breastfeeding-weaned and bottle-fed infants since birth, that suggested only a short and limited benefit from human milk which disappears with the cessation of breastfeeding. ${ }^{22}$ However, the subsequent study was improved that length of breastfeeding is related with decreased clinical duration of bronchiolitis with decreased airway concentration of the neutrophil chemokine interleukin-8. ${ }^{24)}$

Limitation of our retrospective study, we could not obtain the exact proportion and precise duration of BMF in the MF group, and evaluate the family history of allergy and asthma. Weight and age determine the severity of RSV infection; however, we could not explain the reason why infants in the MF group had lower weight and were younger than the infants in the BMF and AMF groups. In our study, the proportion of infants in the MF and AMF (39\%, 37.2\%) groups were larger than the BMF group (22.9\%). This may be reflected in the lower admission rate of the BMF group after RSV infection. ${ }^{4)}$

So far, parents and caregivers did not recognize deeply why baby must be fed with breast milk in Korea. ${ }^{21)}$ Our finding for the positive association of human milk on the oxygen need during RSV infection in infant might have a very important message on the attitude of selecting the feeding type as a real-life data in Korea. When there are no effective treatment regimens yet for RSV infection. Breastfeeding lowers oxygen need, possible ICU care, risk of respiratory distress, and may improve the clinical outcome of RSV infection. Severe early RSV bronchiolitis is associated with an increased prevalence of subsequent recurrent wheezing persisting into early adulthood, reduced spirometer after RSV infection may reflect airway remodeling. ${ }^{9}{ }^{9}$ Breastfeeding could decrease subsequent wheezing similar to the preventive effect of Palivizumab on recurrent wheezing. ${ }^{11)}$

In conclusion, despite of many limitations of retrospective study, oxygen application rate was significantly lower in the BMF group in this study. It is inferred that exclusive breast feed. ing may lower the severity of RSV infection. With our clinical study and referring to other studies that have investigated the immunological benefits of breast milk, breastfeeding should be encouraged to reduce the severity and subsequent complications of RSV infection. Further research should be conducted to compare prospectively the incidence of infections or other diseases in breastfed infants in our country to confirm the excellence of breastfeeding and to increase BRs, especially for infants with proven risk factors such as young age, prematurity, family history of allergy, underlying diseases and older sibling.

\section{Conflicts of interest}

No potential conflict of interest relevant to this article was reported.
Acknowledgments

This research was supported by a fund (KSBM-001\#) by

Research grant of Korean Society of Breastfeeding Medicine.

\section{References}

1. Section on Breastfeeding. Breastfeeding and the use of human milk. Pediatrics 2012;129:e827-41.

2. Cacho NT, Lawrence RM. Innate Immunity and Breast Milk. Front Immunol 2017;8:584.

3. Ip S, Chung M, Raman G, Chew P, Magula N, DeVine D, et al. Breastfeeding and maternal and infant health outcomes in developed countries. Evid Rep Technol Assess (Full Rep) 2007;(153):1-186.

4. Kliegman RM, Stanton BF, St. Geme JW III, Schor NF, Behrman RE, editors. Nelson textbook of pediatrics. 21th ed. Philadelphia (PA): Saunders/Elsevier, 2019.

5. Murray J, Bottle A, Sharland M, Modi N, Aylin P, Majeed A, et al. Risk factors for hospital admission with RSV bronchiolitis in England: a population-based birth cohort study. PLoS One 2014;9:e89186.

6. Law BJ, Carbonell-Estrany X, Simoes EA. An update on respiratory syncytial virus epidemiology: a developed country perspective. Respir Med 2002;96 Suppl B:S1-7.

7. Stein RT, Sherrill D, Morgan WJ, Holberg CJ, Halonen M, Taussig LM, et al. Respiratory syncytial virus in early life and risk of wheeze and allergy by age 13 years. Lancet 1999;354:541-5.

8.Henderson J, Hilliard TN, Sherriff A, Stalker D, Al Shammari N, Thomas HM. Hospitalization for RSV bronchiolitis before 12 months of age and subsequent asthma, atopy and wheeze: a longitudinal birth cohort study. Pediatr Allergy Immunol 2005;16:386-92.

9. Sigurs N, Aljassim F, Kjellman B, Robinson PD, Sigurbergsson F, Bjarnason R, et al. Asthma and allergy patterns over 18 years after severe RSV bronchiolitis in the first year of life. Thorax 2010;65:1045-52.

10. Eiland LS. Respiratory syncytial virus: diagnosis, treatment and prevention. J Pediatr Pharmacol Ther 2009;14:75-85.

11. Mochizuki H, Kusuda S, Okada K, Yoshihara S, Furuya H, Simões EAF, et al. Palivizumab prophylaxis in preterm infants and subsequent recurrent wheezing. six-year follow-up study. Am J Respir Crit Care Med 2017;196:29-38.

12. Saglani S. Viral infections and the development of asthma in children. Ther Adv Infect Dis 2013;1:139-50.

13. Nishimura T, Suzue J, Kaji H. Breastfeeding reduces the severity of respiratory syncytial virus infection among young infants: a multi-center prospective study. Pediatr Int 2009;51:812-6.

14. Chung SH, Kim HR, Choi YS, Bae CW. Trends of breastfeeding rate in Korea (1994-2012): comparison with OECD and other countries. J Korean Med Sci 2013;28:1573-80.

15. Ralston SL, Lieberthal AS, Meissner HC, Alverson BK, Baley JE, Gadomski AM, et al. Clinical practice guideline: the diagnosis, management, and prevention of bronchiolitis. Pediatrics 2014;134:e1474-502.

16. Tang YW, Graham BS. T cell source of type 1 cytokines determines illness patterns in respiratory syncytial virus-infected mice. J Clin Invest 1997;99:2183-91.

17. Carbonell-Estrany X, Fullarton JR, Rodgers-Gray BS, Gooch KL, Vo PG, Figueras-Aloy J. Can we improve the targeting of respiratory syncytial virus (RSV) prophylaxis in infants born 32-35 weeks' gestational age with more informed use of risk factors? J Matern Fetal Neonatal Med 2015;28:1133-41.

18. Roine I, Fernandez JA, Vásquez A, Cáneo M. Breastfeeding reduces immune activation in primary respiratory syncytial virus infection. Eur Cytokine Netw 2005;16:206-10.

19. Chiba Y, Minagawa T, Mito K, Nakane A, Suga K, Honjo T, et al. Effect of breast feeding on responses of systemic interferon and virus-specific lymphocyte transformation in infants with respiratory syncytial virus in. fection. J Med Virol 1987;21:7-14. 
20. Lönnerdal B. Breast milk: a truly functional food. Nutrition 2000;16:50911.

21. Choi EJ. Breastfeeding-related social environmental factors and their policy implications. Sejong (Korea): Health and Welfare Forum, Korea Institute for Health and Social Affairs, 2017:72-81.

22. Dixon DL. The role of human milk immunomodulators in protecting against viral bronchiolitis and development of chronic wheezing illness.
Children (Basel) 2015;2:289-304.

23. Flores P, Guimarães J, Videira Amaral JM. Th1 and th 2 cytokine expression in nasopharyngeal secretions during acute bronchiolitis in child. ren younger than two years old. Allergol Immunopathol (Madr) 2011; 39:3-9.

24. Hussell T, Baldwin CJ, O'Garra A, Openshaw PJ. CD8+ T cells control Th2-driven pathology during pulmonary respiratory syncytial virus infection. Eur J Immunol 1997;27:3341-9. 